\title{
Unbalanced low-density neutron matter
}

\author{
Alexandros Gezerlis* \\ ExtreMe Matter Institute EMMI, GSI Helmholtzzentrum für Schwerionenforschung GmbH, \\ 64291 Darmstadt, Germany \\ Institut für Kernphysik, Technische Universität Darmstadt, 64289 Darmstadt, Germany \\ E-mail: gezerlis@theorie.ikp.physik.tu-darmstadt.de
}

\section{Rishi Sharma}

TRIUMF, 4004 Wesbrook Mall, Vancouver, BC, V6T 2A3, Canada,

E-mail: rishi@triumf.ca

\begin{abstract}
We consider polarized neutron matter at low densities. We have performed Diffusion Monte Carlo simulations for normal neutron matter with different population numbers for each species. We analyze the competition between different phases in the grand canonical ensemble and mention aspects of neutron-star phenomenology that are impacted by the effects described.
\end{abstract}

Xth Quark Confinement and the Hadron Spectrum,

October 8-12, 2012

TUM Campus Garching, Munich, Germany

${ }^{*}$ Speaker. 


\section{Introduction}

Systems which feature $s$-wave pairing between fermions of different species — say $\uparrow$ and $\downarrow$ fermions - exhibit interesting phase structure as we vary the difference in their chemical potentials, $\delta \mu=\left|\left(\mu_{\uparrow}-\mu_{\downarrow}\right) / 2\right|$. For small enough $\delta \mu$, the free energy gain provided by Cooper pairing drives the system into a superfluid state. All fermions are paired up, and the number densities of $\uparrow$ particles $\left(n_{\uparrow}\right)$ and $\downarrow$ particles $\left(n_{\downarrow}\right)$ are equal. As we increase $\delta \mu$, pairing is stressed since in the absence of pairing the corresponding Fermi surfaces would be split and the system would be polarized, $\delta n=n_{\uparrow}-n_{\downarrow} \neq 0$. At large enough $\delta \mu$ the pairing is disrupted and the system exists in a normal phase.

Stressed pairing of this kind shows up in a variety of physical systems. In quark matter at densities relevant to the phenomenology of neutron stars, the strange quark mass gives rise to a splitting between the effective quark chemical potentials [2]. In cold atoms, polarization can be created by trapping different numbers of fermions of the two hyperfine states that pair. Since the number of particles in the traps is fixed, polarization leads to phase separation [3] which can be observed [4] by looking at their density profile.

In these proceedings, we will focus on the pairing between spin $\uparrow$ and $\downarrow$ neutrons in the inner crust of neutron stars. Dipole interactions with the magnetic field split the chemical potentials of the two. At a critical value of the field, pairing breaks, and there is a transition to the normal phase from the superfluid phase.

Calculating the critical field is relevant for the phenomenology of neutron-star crusts because the transport properties of the two phases are very different. The superfluid phase can transport heat efficiently via Goldstone bosons (although mixing between the superfluid Goldstone bosons and the lattice phonons suppresses this effect [5,6]). Furthermore, the normal phase features gapless neutrons near the Fermi surface, and consequently has a large specific heat. Both these effects tend to increase the time scales [7] associated with heat transport in the inner crust if a significant fraction of the crustal neutrons are in the normal phase.

The phase diagram as a function of $\delta \mu$ is well understood in BCS theory. The normal phase is favored over the superfluid phase for $\delta \mu=\delta \mu_{c}>\Delta_{0} / \sqrt{2}$ where $\Delta_{0}$ is the pairing gap for $\delta \mu=0$. In a small window near $\delta \mu_{c}$ exotic phases called LOFF [8] phases are theoretically favored.

Similar results can be argued for "weak coupling" [9]: a regime where the separation between the particles is much greater than the scattering length. More formally, defining $k_{F}=\left(3 \pi^{2}\left(n_{\uparrow}+\right.\right.$ $\left.\left.n_{\downarrow}\right)\right)^{1 / 3}$, and the scattering length as $a$, the free energy of the normal and the superfluid phases can be written in terms of functions that become smaller as $\left|k_{F} a\right|$ decreases, if $k_{F} a$ is small.

For strongly coupled Fermi systems, however, we don't know of a controlled expansion technique to calculate thermodynamic properties. Therefore, one needs to perform intensive numerical calculations to compute the energies of both the normal and the superfluid phase.

In these proceedings we will present QMC calculations of the energy of the competing phases for three number densities $n_{1}=6.65 \times 10^{-4} \mathrm{fm}^{-3}, n_{2}=2.16 \times 10^{-3} \mathrm{fm}^{-3}$, and $n_{3}=5.32 \times 10^{-3}$ $\mathrm{fm}^{-3}$ for different polarizations. For comparison, these correspond respectively to $0.0042 n_{\text {sat }}$, $0.0135 n_{\text {sat }}$, and $0.033 n_{\text {sat }}$ where $n_{\text {sat }}=0.16 \mathrm{fm}^{-3}$ is the saturation density. The neutron-neutron

\footnotetext{
${ }^{1}$ Here we are assuming that the masses of the two species are equal. A similar stress arises in systems of fermions with different masses (for eg. [1]).
} 


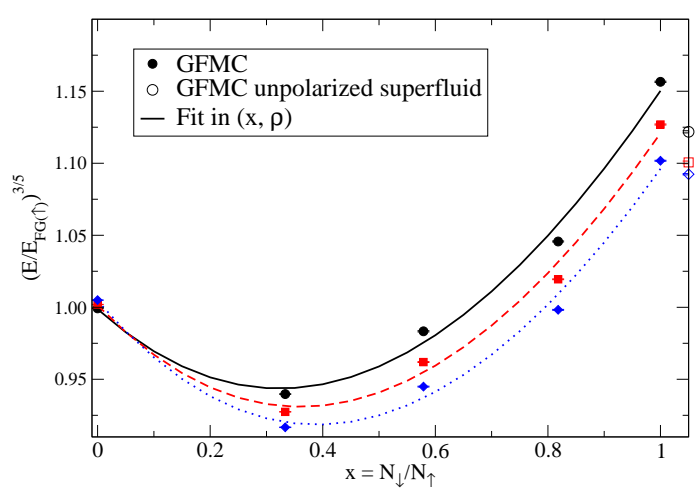

Figure 1: Ground-state energy per particle (in units of the free Fermi gas energy) scaled to the power of 3/5 for normal spin-polarized neutron matter. Shown are QMC results at three different total densities $n_{1}, n_{2}, n_{3}$ (as points: black circles, red squares, blue diamonds, respectively), along with fits to the Monte Carlo results using the set of functions of $(x, \rho)$ given in Eq. 3.6. (as lines: black solid, red dashed, blue dotted, respectively). Also shown using hollow symbols are the results for an unpolarized superfluid.

interaction has a large scattering length $(a \simeq-18.6 \mathrm{fm})$ compared to the effective range $r_{e}, \sim 2.7$ $\mathrm{fm}$. We see that at the densities we consider $k_{F} a$ is large in magnitude $\left(k_{F} a=5.02,7.44\right.$, and 10.05 corresponding to the three densities which are all deep in the strongly coupled regime). For reference, we note that $k_{F} r_{e}=0.59,0.88$, and 1.19 .

Phase competition is analyzed very simply in the grand canonical ensemble. If we know the pressure of the competing phases as a function of the average chemical potential $\mu=\left(\mu_{\uparrow}+\mu_{\downarrow}\right) / 2$ and the splitting $\delta \mu$, then the phase with the larger pressure at given $\mu$ wins. At the phase transition the pressure and $\mu$ should match. ( $\delta \mu$ need not be the same between coexisting phases, as long as $\delta \mu<\Delta$, where $\Delta$ is the superfluid gap.)

The direct evaluation of the pressure as a function of $\mu$ is very difficult because of the sign problem. For a fixed $n_{\uparrow}, n_{\downarrow}$ a creative technique known as fixed node QMC [10] has been developed to calculate the energy of fermionic systems $\mathscr{E}(n, \delta n)$ accurately. We will describe this calculation next.

\section{Calculation of $\mathscr{E}$}

The heart of the calculation is the accurate evaluation of the energy as a function of density and polarizations $[11,12]$. We use the Diffusion Monte Carlo (DMC) method which is expected to be exact, modulo the fermion-sign problem. Schematically, this entails taking a variational wave function $\Psi_{V}$ that encodes knowledge about the physics of the system (e.g. is it superfluid, does it contain trimers, etc.). We first perform a Variational Monte Carlo simulation and then project out the exact, lowest-energy eigenstate $\Psi_{0}$ from the trial (variational) wave function $\Psi_{V}$. In other words, we treat the Schrödinger equation as a diffusion equation in imaginary time and evolve the variational wave function up to the limit of $\tau \rightarrow \infty$. This can be seen by expanding $\Psi_{V}$ in terms of 
the complete set $\Psi_{i}$ of the exact eigenstates of the Hamiltonian (with eigenvalues $E_{i}$ ):

$$
\begin{aligned}
\Psi(\tau) & =e^{-\left(H-E_{T}\right) \tau} \Psi_{V}=\sum_{i} \alpha_{i} e^{-\left(E_{i}-E_{T}\right) \tau} \Psi_{i} \\
& =\alpha_{0} e^{-\left(E_{0}-E_{T}\right) \tau} \Psi_{0}, \quad \lim \tau \rightarrow \infty
\end{aligned}
$$

where $E_{T}$ is called the trial energy. In practice, the DMC technique is implemented by discretizing $\tau$.

As already implied, the trial wave function is critically important, in that it is used as the fixednode constraint. In addition, it is commonly also used as an importance-sampling function. In this problem, we are interested in simulating non-superfluid systems with unequal populations of spin-up and spin-down neutrons. Thus, modulo a conceptually irrelevant (but numerically useful) Jastrow term, the wave function describes the particles as being in a free Fermi gas (two, to be precise, one for each spin-orientation). This formalism localizes all the correlations within the Jastrow functions, leaving the rest of the wave function as a product of two Slater determinants:

$$
\Phi_{S}(\mathbf{R})=\mathscr{A}\left[\phi_{n}\left(r_{1}\right) \phi_{n}\left(r_{2}\right) \ldots \phi_{n}\left(r_{N_{\uparrow}}\right)\right] \mathscr{A}\left[\phi_{n}\left(r_{1^{\prime}}\right) \phi_{n}\left(r_{2^{\prime}}\right) \ldots \phi_{n}\left(r_{N_{\downarrow}^{\prime}}\right)\right]
$$

where the unprimed (primed) indices correspond to spin-up (spin-down) neutrons, $N_{\uparrow}+N_{\downarrow}^{\prime}=N$, $\mathscr{A}$ is the antisymmetrizer, and the $\phi_{n}\left(r_{k}\right)$ are plane waves with periodic boundary conditions inside a box of volume $L^{3}$. Using real numbers is often preferable numerically, so it is customary to use linear combinations of the eigenfunctions. Shown below is the result for 7 particles:

$$
\left|\begin{array}{cccc}
1 & 1 & \ldots & 1 \\
\cos \left(2 \pi x_{1} / L\right) & \cos \left(2 \pi x_{2} / L\right) & \ldots & \cos \left(2 \pi x_{7} / L\right) \\
\cos \left(2 \pi y_{1} / L\right) & \cos \left(2 \pi y_{2} / L\right) & \ldots & \cos \left(2 \pi y_{7} / L\right) \\
\cos \left(2 \pi z_{1} / L\right) & \cos \left(2 \pi z_{2} / L\right) & \ldots & \cos \left(2 \pi z_{7} / L\right) \\
\sin \left(2 \pi x_{1} / L\right) & \sin \left(2 \pi x_{2} / L\right) & \ldots & \sin \left(2 \pi x_{7} / L\right) \\
\sin \left(2 \pi y_{1} / L\right) & \sin \left(2 \pi y_{2} / L\right) & \ldots & \sin \left(2 \pi y_{7} / L\right) \\
\sin \left(2 \pi z_{1} / L\right) & \sin \left(2 \pi z_{2} / L\right) & \ldots & \sin \left(2 \pi z_{7} / L\right)
\end{array}\right|
$$

The number 7 was chosen because it corresponds to a closed shell. Closed shells exhibit no ambiguity in the selection of the momenta different particles are placed in.

In our simulations of polarized normal neutron matter we used $33+0,57+19,57+33,33+$ 27 , and $33+33$ particles. These correspond to relative fractions of $x=0,0.333,0.579,0.818$, and 1 , respectively. At each particle number combination, we performed simulations for three distinct total number densities $\left[n=\left(N_{\uparrow}+N_{\downarrow}\right) / L^{3}\right]$, as mentioned earlier. The results of these computations are given in Fig. 1, which shows the values of

$$
g(x, n)=\left(\frac{\mathscr{E}(x, n)}{\mathscr{E}_{F G(\uparrow)}}\right)^{3 / 5}
$$

where $\mathscr{E}_{F G(\uparrow)}=\frac{3}{5} \frac{\left(6 \pi^{2}\right)^{2 / 3}}{2 m}\left(n_{\uparrow}\right)^{5 / 3}$ is the energy of a non-interacting gas of a single species. 


\section{The free energy functions}

\subsection{The unpolarized superfluid phase}

The energy and the gap for the unpolarized superfluid phase for $n_{1}, n_{2}$ and $n_{3}$ were calculated in $[13,14]$. A convenient parameterization for the data can be obtained by considering the dimensionless function $g_{S F}(n)$, defined by the relation

$$
\mathscr{E}(n)=\frac{3}{10} \frac{\left(3 \pi^{2}\right)^{2 / 3}}{2 m}\left(n g_{S F}(n)\right)^{5 / 3},
$$

where, $\mathscr{E}$ is the energy density. The prefactors and the power of $n$ in the definition is chosen so that for a system of non-interacting fermions, $g=1$. Since $g_{S F}$ is dimensionless, the density dependence in $g_{S F}$ can only arise in appropriate combinations with $a$ and $r_{e}$. In particular, it motivates considering the form

$$
g_{S F}(n)=g_{0,0}+g_{0,1} r_{e} n^{1 / 3}+g_{1,0} \frac{1}{n^{1 / 3} a}+\cdots
$$

For the relevant densities, a good fit is obtained for $g_{0,0}=0.942, g_{0,1} r_{e}=0.003 \mathrm{MeV}^{-1}, g_{1,0} / a=$ $2.301 \mathrm{MeV}$. For comparison, for the unitary Fermi gas $\left(a \rightarrow \infty, r_{e} \rightarrow 0\right), g_{S F}$ is independent of the density and is given by $(2 \xi)^{3 / 5}$, where $\xi$ is the Bertsch parameter. For $\xi=0.38$ [15] calculated using similar techniques, $(2 \xi)^{3 / 5}=0.848$, which is close to $g_{0,0}$. It is not surprising that $g_{0,0}$ differs from the unitary value since this calculation is being done for neutron matter, and the interaction is more complicated. One expects deviations also because the largest $k_{F} r_{e}$ is of the order of 1 .

With this parameterization, it is easy to calculate the pressure as a function of the chemical potential using standard thermodynamical relations.

\subsection{The normal phase}

The analysis of the normal phase is a little more involved because the energy depends both on the density $n$ and the relative fraction $x=n_{\downarrow} / n_{\uparrow}$ where we take $n_{\uparrow}$ to be the majority species. A convenient generalization of Eq. 3.1 is

$$
\mathscr{E}(x, n)=\frac{3}{5} \frac{\left(6 \pi^{2}\right)^{2 / 3}}{2 m}\left(n_{\uparrow} g(x, n)\right)^{5 / 3},
$$

which is equivalent to Eq. 2.4. The chemical potential is:

$$
\mu=\left.\frac{\partial \mathscr{E}}{\partial n}\right|_{\delta n}=\frac{3^{2 / 3} \pi^{4 / 3}\left(\frac{n}{x+1}\right)^{2 / 3} g(x, n)^{2 / 3}\left(\frac{2 n}{x+1} \frac{\partial g}{\partial n}(x, n)+(1-x) \frac{\partial g}{\partial x}(x, n)+g(x, n)\right)}{2^{4 / 3} m} .
$$

The chemical potential splitting is:

$$
\delta \mu=\left.\frac{\partial \mathscr{E}}{\partial \delta n}\right|_{n}=\frac{3^{2 / 3} \pi^{4 / 3}\left(\frac{n}{x+1}\right)^{2 / 3} g(x, n)^{2 / 3}\left((-x-1) \frac{\partial g}{\partial x}(x, n)+g(x, n)\right)}{2^{4 / 3} m} .
$$

We fit the values of $g$ given in Fig. 1 with a linear combination of the following functions,

$$
\left\{(x+1), \frac{(x+1)}{\rho^{1 / 3}},(x+1) \rho^{1 / 3},(x+3)^{2}, \frac{(x+3)^{2}}{\rho^{1 / 3}},(x+3)^{2} \rho^{1 / 3},(x+5)^{3}, \frac{(x+5)^{3}}{\rho^{1 / 3}},(x+5)^{3} \rho^{1 / 3}\right\}
$$

The $x$ dependence is chosen so that at $\delta \mu=0$ for $x=1$.

The pressure is simply $P=-\mathscr{E}(n, \delta n)+\mu n+\delta \mu \delta n$. 


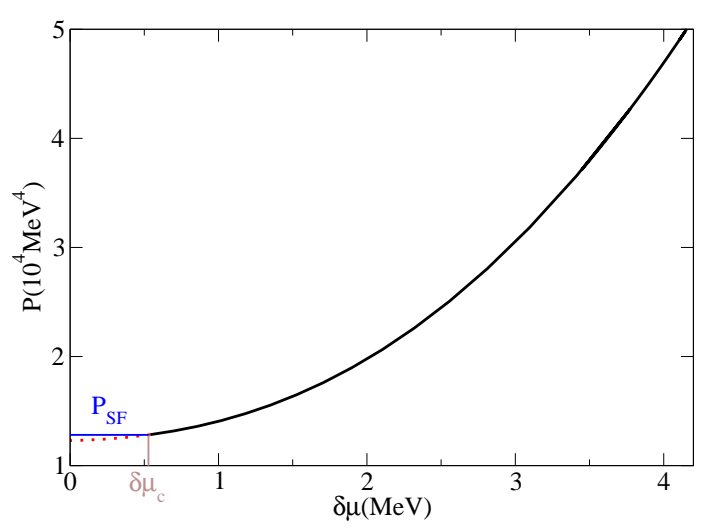

Figure 2: Pressure as a function of $\delta \mu$ for fixed $\mu=1.94 \mathrm{MeV}$. For $\delta \mu>\delta \mu_{c}=0.53 \mathrm{MeV}$ the unpaired phase (bold black curve) has a higher pressure. At $\delta \mu_{c}$ the $S F$ and the $N P$ phases can coexist and for smaller $\delta \mu$, the $S F$ phase is favored. On the other extreme for $\delta \mu=4.18 \mathrm{MeV}, x=0.0005$. We choose $\mu$ so that the density of the normal phase at $\delta \mu_{c}$ is $n_{2}$.

\section{Phase competition}

For a given $\mu, \delta \mu_{c}$ is simply the point where the pressure curves of the unpolarized superfluid and the normal phases cross. For example, from the plot of the pressure as a function of $\delta \mu$ for $\mu=1.94 \mathrm{MeV}$ in Fig. 2 we conclude that $\delta \mu_{c}=0.53 \mathrm{MeV}$.

In Fig. 3 we show a plot of $\delta \mu_{c} / E_{F}$ as a function of $\mu$, where $E_{F}=k_{F}^{2} /(2 m)$ ( $m$ is the mass of the neutron and $k_{F}$ is the Fermi momentum corresponding to the density in the superfluid phase). The range of $\mu$ is chosen such that the density of the superfluid varies from $n_{1}$ at the smallest value to $n_{3}$ at the largest. For reference we note that $\Delta / E_{F}$ increases gradually from 0.4 at $n_{1}$ to 0.44 at $n_{3}$ [13]. This can be seen as a competition between stronger pairing as $\left|k_{F} a\right|$ increases weakened by increasing $k_{F} r_{e}$. The effect of the effective range on $\delta \mu_{c} / E_{F}$ is much more pronounced. We see that, not surprisingly, this makes it "easier" (in a dimensionless sense) to break Cooper pairing at higher density. The two curves give an estimate of the uncertainty associated with different interpolating functions of the same data.

\section{Conclusions}

To summarize, we have performed Diffusion Monte Carlo simulations for polarized neutron matter at low density. Low density implies that the interaction between neutrons is greatly simplified: three-body forces and spin-orbit $\&$ tensor interactions have a minimal effect, leading to a mainly s-wave interaction. We have included the s-wave channel of the high-quality phenomenological Argonne v18 potential, along with perturbative p-wave corrections. The computations we have performed were undertaken for normal neutrons of varying population numbers and are expected to provide tight upper bounds to the true ground-state energies.

Using these results we have extracted the critical chemical potential splitting $\delta \mu_{c}$ at which there is a transition from the superfluid state to the normal state as we increase $\delta \mu$. 


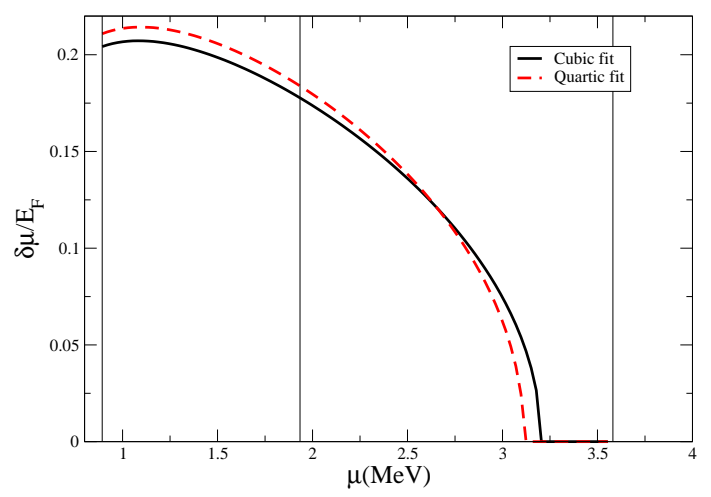

Figure 3: Plot of $\delta \mu / E_{F}$ as a function of $\mu$. The two curves correspond to two choices of interpolating functions in $x$. The solid line (black) corresponds to the set of functions given in Eq. 3.6, up to cubic power in $x$. The dashed line (red) corresponds to a larger set including quartic powers. The vertical lines at increasing $\mu$ correspond to the chemical potentials at which the densities of the unpolarized superfluid phase are $n_{1}, n_{2}$ and $n_{3}$ respectively.

For a system with unequal number densities of $\uparrow$ and $\downarrow$ neutrons our calculations imply that the energetically favored state is that of phase separation into an unpolarized superfluid and a polarized normal neutron gas. This situation directly parallels what is found in systems of ultracold fermionic atoms [16] which have no effective-range and p-wave contributions. The cold-atom system has also been probed experimentally and quantitatively well-described by QMC calculations, lending credence to the claim that our results for neutron matter are also quite accurate. Using our simulations and worked published earlier, we have extracted the critical relative fractions above which phase separation occurs (for details see [11]). For relative fractions above the critical values the competing phase that has been considered [12] is a homogeneous polarized superfluid state. The mixed phase is energetically favored compared to the polarized superfluid state, meaning that we have provided the lowest-energy configuration to date for the system under study. At relative fractions lower than the critical value, our analysis suggests that the system is entirely normal.

The quenching of pairing, if it materializes in a neutron star sufficiently magnetized to polarize the neutrons, would have directly observable consequences, as superfluidity impacts the specific heat and more generally the thermal behavior of the star. This would also hold even for slightly smaller magnetic fields. More generally, our predictions are in principle also relevant to experiments with cold atoms which could in the future be performed for finite effective ranges in the laboratory. [17] Such experiments today make use of unequal spin populations as a matter of course. Importantly, our microscopic simulations can also impact the terrestrial phenomenology of nuclei. Skyrme-family energy-density functional theories of nuclei have traditionally been fitted to experimentally measured masses of nuclei, while also sometimes including infinite matter constraints. The latter in the past consisted of the behavior of nuclear matter, while more recently the neutron-matter pairing gap has also been used as an extra constraint. [18, 19] Following this path to its logical consequences, a recently completed work [20] directly compares the neutron polaron binding energy (i.e. the binding of one spin-down impurity embedded in a sea of spin- 
up neutrons) with the predictions following from a group of established Skyrme parametrizations. Extending this line of thinking, one could generalize such comparisons to finite relative fractions and thus provide a further constraint to nuclear density functionals: this all follows from the fact that our DMC results provide a dependable microscopic solution of the Schroedinger equation. Neutron-rich nuclei, in particular, are expected to be impacted by the physics of polarized neutron matter.

There are many ways in which one could build on the calculations discussed in this contribution. One could add an external periodic potential, which would stand for the periodic lattice of neutron-rich nuclei found in a neutron-star crust and could also teach us something about surface effects in neutron drops. The physics of the static response of polarized (or, for that matter, unpolarized) neutron matter is wholly uncharted from a microscopic perspective. Any predictions on this system could also be extended to the relevant setting of optical-lattice experiments, which could indirectly teach us something about the neutron case, even without an effective range. Similarly, one could extend calculations such as these to more species or even finite-temperature effects, in an effort to directly probe thermal effects in neutron-star crusts and cores.

\section{References}

[1] A. Gezerlis, S. Gandolfi, K. E. Schmidt, and J. Carlson, Phys. Rev. Lett. 103, 060403 (2009)

[2] M. Alford, J. Berges, and K. Rajagopal, Nucl. Phys. B 558, 219 (1999).

[3] P. F. Bedaque, H. Caldas, and G. Rupak, Phys. Rev. Lett. 91, 247002 (2003).

[4] Y. Shin et al., Phys. Rev. Lett. 97, 30401 (2006).

[5] V. Cirigliano, S. Reddy, and R. Sharma, Phys. Rev. C 84, 045809 (2011).

[6] N. Chamel, D. Page, and S. Reddy, arXiv:1210.5169 (2012).

[7] E. F. Brown and A. Cumming, Astrophys. J. 698, 1020 (2009).

[8] A.I. Larkin and Y.N. Ovchinnikov, Sov. Phys. JETP,762,20,1965 and Fulde, Peter and Ferrell, Richard A., Phys. Rev.,3A,1964.

[9] R. Sharma and S. Reddy, Phys. Rev. A78, 063609 (2008).

[10] J. Carlson, J. Morales, Jr., V. R. Pandharipande, and D. G. Ravenhall, Phys. Rev. C 68, 025802 (2003).

[11] A. Gezerlis and R. Sharma, Phys. Rev. C 85, 015806 (2012)

[12] A. Gezerlis, Phys. Rev. C 83, 065801 (2011).

[13] A. Gezerlis and J. Carlson, Phys. Rev. C 77, 032801(R) (2008).

[14] A. Gezerlis and J. Carlson, Phys. Rev. C 81, 025803 (2010).

[15] M. M. Forbes, S. Gandolfi, and A. Gezerlis, Phys. Rev. Lett., 106, 235303, (2011).

[16] S. Pilati and S. Giorgini, Phys. Rev. Lett. 100, 030401 (2008).

[17] B. Marcelis, B. Verhaar, and S. Kokkelmans, Phys. Rev. Lett. 100, 153201 (2008).

[18] N. Chamel, S. Goriely, and J.M. Pearson, Nucl. Phys. A812, 72 (2008).

[19] M. Dutra et al., Phys. Rev. C 85, 035201 (2012).

[20] M. M. Forbes, A. Gezerlis, K. Hebeler, T. Lesinski, and A. Schwenk, submitted to Phys. Rev. Lett. (2013). 